OPEN ACCESS

Edited by:

Mitsugu Fujita,

Kindai University Faculty of Medicine,

Japan

Reviewed by:

Takaki Miyata,

Nagoya University, Japan Siu Ping Ngok,

Stanford University School of

Medicine, USA

*Correspondence:

Takaaki Matsui,

Gene Regulation Research, Nara Institute of Science and Technology, 8916-5 Takayama, Ikoma 630-0101,

Nara, Japan

matsui@bs.naist.jp

Specialty section:

This article was submitted to

Cell Adhesion and Migration,

a section of the journal

Frontiers in Cell and Developmental

Biology

Received: 16 March 2015 Accepted: 22 April 2015

Published: 07 May 2015

Citation:

Matsui T, Ishikawa $\mathrm{H}$ and Bessho $Y$ (2015) Cell collectivity regulation within migrating cell cluster during Kupffer's vesicle formation in zebrafish

Front. Cell Dev. Biol. 3:27.

doi: 10.3389/fcell.2015.00027

\section{Cell collectivity regulation within migrating cell cluster during Kupffer's vesicle formation in zebrafish}

\author{
Takaaki Matsui *, Hiroshi Ishikawa and Yasumasa Bessho \\ Gene Regulation Research, Nara Institute of Science and Technology, Nara, Japan
}

Although cell adhesion is thought to fasten cells tightly, cells that adhere to each other can migrate directionally. This group behavior, called "collective cell migration," is observed during normal development, wound healing, and cancer invasion. Loss-of-function of cell adhesion molecules in several model systems of collective cell migration results in delay or inhibition of migration of cell groups but does not lead to dissociation of the cell groups, suggesting that mechanisms of cells staying assembled as a single cell cluster, termed as "cell collectivity," remain largely unknown. During the formation of Kupffer's vesicle (KV, an organ of laterality in zebrafish), KV progenitors form a cluster and migrate together toward the vegetal pole. Importantly, in this model system of collective cell migration, knockdown of cell adhesion molecules or signal components leads to failure of cell collectivity. In this review, we summarize recent findings in cell collectivity regulation during collective migration of KV progenitor cells and describe our current understanding of how cell collectivity is regulated during collective cell migration.

Keywords: collective cell migration, cell adhesion, Kupffer's vesicle, cell signaling, zebrafish

\section{Introduction}

Individual cells have the potential to migrate randomly. When chemo-attractants are distributed in an environment, the cells can migrate toward the attractants. In some situations, cell aggregates can move directionally while maintaining cell adhesions in a process called collective cell migration. Collective cell migration is essential for the generation of basic organ structures such as sheets, clusters, spheres, sprouts, and vesicles in the morphogenetic processes of animal development (Rorth, 2009, 2012; Weijer, 2009; Reig et al., 2014), and is also observed in wound closure and cancer invasion (Friedl and Gilmour, 2009; Friedl et al., 2012). Collective cell migration shares some features with individual cell migration but also has unique ones. These features have been described in several in-depth reviews cited in this review (Friedl and Gilmour, 2009; Rorth, 2009, 2012; Weijer, 2009; Friedl et al., 2012; Reig et al., 2014).

Loss-of-function of chemo-attractants, their receptors, signal mediators, and cell adhesion molecules blocks or delays the directed migration of the cell groups in model systems of collective cell migration such as zebrafish lateral lines and Drosophila border cells (Niewiadomska et al., 1999; Kerstetter et al., 2004; Wilson et al., 2007; Friedl and Gilmour, 2009; Rorth, 2009; Reig et al., 2014). However, these manipulations unexpectedly does not dissociate the cell groups, suggesting that how "cell collectivity," a feature in which cells stay assembled as a single cell cluster, is produced and maintained within a migrating cell cluster remains largely unknown.

Kupffer's vesicle (KV) is a key organ required for the left-right asymmetric patterning in zebrafish (Amack and Yost, 2004; Essner et al., 2005; Matsui and Bessho, 2012). During KV 
organogenesis, 20-30 KV progenitors called dorsal forerunner cells (DFCs) make a single cluster and migrate together, so $\mathrm{KV}$ organogenesis is represented as one of the model systems of collective cell migration. In our and other's studies, loss-offunctional situations of genes/signals have led to a breaking up of the cluster of KV progenitors (DFCs) without affecting their directed migration (Matsui and Bessho, 2012). We therefore feel that KV organogenesis is a good model for investigating the regulatory mechanisms of cell collectivity formation. In this review, we describe our recent understanding of how cell collectivity is generated and maintained during collective DFC migration.

\section{Review}

\section{KV Formation and Function}

In zebrafish, DFCs first appear in the dorsal site adjacent to the embryonic margin at $6 \mathrm{~h}$ post-fertilization (hpf) (Figure 1) (Essner et al., 2002, 2005; Oteiza et al., 2008; Matsui and Bessho, 2012). About 20-30 DFCs form a single cluster that migrates toward the vegetal pole accompanied by epiboly movement by 10 hpf (Cooper and D’Amico, 1996; Melby et al., 1996). During migration, the DFC cluster undergoes compaction and changes into a bottled shaped cluster. Around the late-gastrulation stage (8hpf), DFCs start to polarize so that multiple focal points are generated within the cluster (Cooper and D'Amico, 1996; Melby et al., 1996; Amack et al., 2007). These points are then rearranged into a single focal point that will expand and become a vesicle lumen by $12 \mathrm{hpf}$. At the same time, motile monocilia are generated on the apical membrane facing the lumen. In the $\mathrm{KV}$, rotation of motile cilia generates a counterclockwise flow of fluid, called nodal flow, and leads to the establishment of leftright asymmetry in the body (Essner et al., 2005; Amack et al., 2007; Oteiza et al., 2008). Several excellent reviews have already described the molecular and cellular mechanisms of left-right patterning and ciliogenesis (Hirokawa et al., 2006, 2012; Ishikawa and Marshall, 2011; Nakamura and Hamada, 2012; Blum et al., 2014; Choksi et al., 2014), so we do not go into those here. Instead, we focus on cell collectivity regulation occurring within a migrating cell cluster.

\section{Cell Adhesive Interactions Formed in a Migrating DFC Cluster}

When DFCs appear as a single cluster at the mid-gastrulation stage (6hpf), cadherin1 and cadherin2 (E-cadherin and $\mathrm{N}$-cadherin in zebrafish, respectively) are expressed in DFCs (Babb et al., 2001; Babb and Marrs, 2004; Esguerra et al., 2007; Harrington et al., 2007; Warga and Kane, 2007; Matsui et al., 2011), suggesting that cadherin-based adherens junction mediates DFC-DFC interaction (Figure 1). Although, in general, formation of adherens junction leads to generation of tight junction (Hartsock and Nelson, 2008), tight junction is not formed between DFCs at $6 \mathrm{hpf}$. Instead, tight junction is formed between a subset of DFCs and overlying enveloping layer

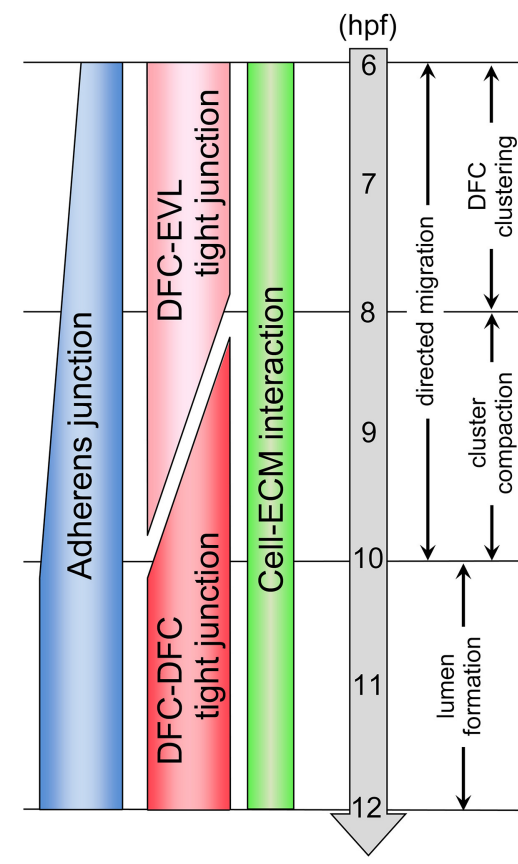

FIGURE 1 | Cell adhesion status during KV organogenesis. KV organogenesis is divided into four steps. First, 20-30 DFCs form a cluster through the formation of adherens junction. A subset of DFCs binds to EVL through the formation of tight junction. Second, the cluster becomes compact. Multiple focal points that enrich tight

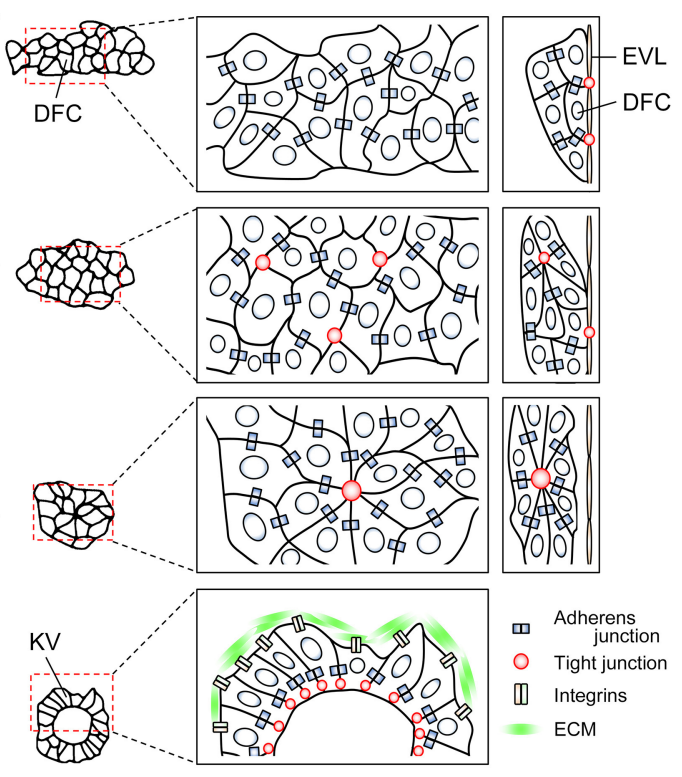

junction components are generated between some DFCs. Third, these points are then rearranged into a single focal point. Fourth, an internal lumen is generated. During collective DFC migration, adherens junction, tight junction, and cell-ECM interaction are generated in a migrating DFC clusters. 
(EVL) cells (Figure 1) (Oteiza et al., 2008). In addition, it has been reported that integrin $\alpha 5$ and integrin $\beta 1 b$ are expressed in migrating DFC cluster at $6 \mathrm{hpf}$ (Ablooglu et al., 2010). These findings suggest that multiple types of cell adhesions including adherens junctions, tight junctions, and cell-extracellular matrix (ECM) interactions are generated in DFCs at the onset of DFC clustering.

During epiboly (6-10 hpf), cadherin1 expression increases in migrating DFCs (Figure 1) (Babb et al., 2001; Babb and Marrs, 2004; Esguerra et al., 2007; Matsui et al., 2011), suggesting that a cadherin1-based adherens junction contributes to the compaction and shape change of the cluster. Within the bottled shaped cluster seen in $8-10 \mathrm{hpf}$, focal points that enrich the tight junction components are generated, and then multiple focal points are rearranged into a single focal point that will be an internal vesicle lumen (Figure 1) (Amack et al., 2007; Oteiza et al., 2008, 2010; Siddiqui et al., 2010). On the other hand, DFC-EVL tight junction is maintained by the end of epiboly (10 hpf) (Figure 1) (Oteiza et al., 2008, 2010; Siddiqui et al., 2010). This means that there is a transition of tight junction formation from DFC-EVL to DFC-DFC pairs during collective DFC migration (Figure 1). However, how the tight junction formation is regulated and how this transition occurs remain unexplored.

\section{Regulatory Mechanisms of Cell Collectivity Formation in the DFC Cluster}

When cadherin1 is knocked down specifically in DFCs, the DFC cluster is broken up into multiple groups of cells at $60-80 \%$ epiboly stages (6.5-8.5 hpf) (Matsui et al., 2011); the "brokenup DFC cluster phenotype" is represented as a sign of failures of cell collectivity (Table 1). This phenotype eventually results in formation of small $\mathrm{KV}$ and randomization of left-right patterning (this means the loss of $\mathrm{KV}$ function), indicating that cell collectivity regulation is required for formation of a functional $\mathrm{KV}$. We also find that strengthening a cadherin1-based adherens junction by cadherin1 overexpression induces the formation of a highly compacted oval-shaped DFC cluster, leading to abnormal formation of KV and loss of KV function (Matsui et al., 2011). These observations suggest that KV formation becomes abnormal in either weak or strong cadherin1-based adherens junctions. This may be a common feature of collective migration because it has been reported that both up- and down-regulation of DE-cadherin induce the delayed collective migration of the border cell cluster during Drosophila oogenesis (Niewiadomska et al., 1999; Schober et al., 2005).

These findings suggest that a fine-tuning of cadherin1 expression and function into a proper range is required for generating proper DFC collectivity. Our recent study has revealed a part of this mechanism: specifically, we characterized a fibroblast growth factor (FGF) positive feedback regulator canopy1 in zebrafish and found that FGF-signal dependent regulation of cadherin1 expression is required for generating proper cell collectivity in the DFC cluster (Matsui et al., 2011). fgf8a secreted from DFCs binds to and activates FGF receptor 1 (fgfr1) in DFCs. The intracellular signal pathway (Ras-Erk pathway) induces canopy1 expression. canopyl with chaperones enhances fgfr1 protein folding within endoplasmic reticulum and increases mature fgfr 1 at the membrane of DFCs, resulting in much higher FGF/Erk signal activity in DFCs. The potentiated FGF/Erk signal induces the expression of cadherin1 via transcriptional activation of $t b x 16$ (Table 1). Furthermore, it has been reported that Nodal signaling regulates the expression of cadherin1 in DFCs via repression of snail1 expression (Table 1) (Esguerra et al., 2007), which is known as a transcriptional repressor of E-cadherin expression in several animals (Batlle et al., 2000; Cano et al., 2000; Hajra et al., 2002).

In addition to these transcriptional regulations of cadherin1, the post-translational regulations of cadherin 1 contribute to the fine-tuning of cell collectivity. The lateral giant larvae 2 (lgl2), one of the lateral giant larvae genes initially identified as tumor suppressor genes in Drosophila, is expressed in DFCs (Tay et al., 2013). $\lg 12$ in cooperation with rab1lb GTPase enhances the trafficking of cadherin1 proteins into a lateral membrane to generate KV lumen, suggesting that regulation of the membrane trafficking of cadherin 1 protein is required for ensuring cell collectivity (Table 1). Heterotrimeric G proteins of the $G \alpha_{12}$ family $\left(G \alpha_{12}\right.$ and $\left.G \alpha_{13}\right)$ bind to the intercellular domain of cadherin 1 and interfere with the link between cadherin1 and $\beta$-catenin, leading to the inhibition of cell-cell adhesion (Lin et al., 2009). Importantly, loss- and gain-of-function of the $\mathrm{G} \alpha_{12}$ family in zebrafish result in a breaking up of the DFC cluster into small groups of cells (Table 1) (Lin et al., 2009), suggesting again that fine-tuning of the cadherin1 expression is required for generating cell collectivity. Taken together, these results suggest that the cadherin1-based adherens junction is regulated by both transcriptional and post-translational mechanisms and that these fine-tuning systems are essential for generating proper DFC collectivity during collective DFC migration.

The DFC-specific knockdown of integrin $\alpha 5$ or integrin $\beta 1 b$ results in the breaking up of the DFC cluster (Ablooglu et al., 2010), demonstrating that cell-ECM interaction contributes to DFC collectivity (Figure 1 and Table 1 ). integrin $\alpha 5 / \beta 1 \mathrm{~b}$ is known to recognize RGD-peptide containing ligands (e.g., fibronectin, vitronectin, and osteopontin). Although overexpression of a dominant negative form of fibronectin and fibronectin mutations, including natter, leads to the randomization of left-right asymmetric body plan and cardia bifida, respectively (Trinh and Stainier, 2004; Compagnon et al., 2014), it has not been reported that DFC collectivity is compromised in embryos injected with the dominant negative form of fibronectin and fibronectin mutants. Although laminin- $\alpha 1 \beta 1 \gamma 1$ accumulate around $\mathrm{KV}$ and DFC-specific knockdown of laminin- $\gamma 1$ results in ciliogenesis failures and the randomization of a left-right asymmetric body plan (Compagnon et al., 2014), this manipulation does not lead to the breaking up of the DFC cluster. Therefore, it is unlikely that fibronectin and laminins are a ligand for integrin $\alpha 5 / \beta 1 \mathrm{~b}$ in migrating DFCs. Additional experiments are required to identify the ligand(s) for integrin $\alpha / \beta 1 \mathrm{~b}$ during collective DFC migration in the near future.

As stated above, loss-of-function of cadherin1-based adherens junction or integrin $\alpha 5 / \beta 1 \mathrm{~b}-\mathrm{ECM}$ interaction in DFCs leads to a broken up DFC cluster (Table 1) (Ablooglu et al., 2010; Matsui et al., 2011). However, some DFCs bind to each other even 

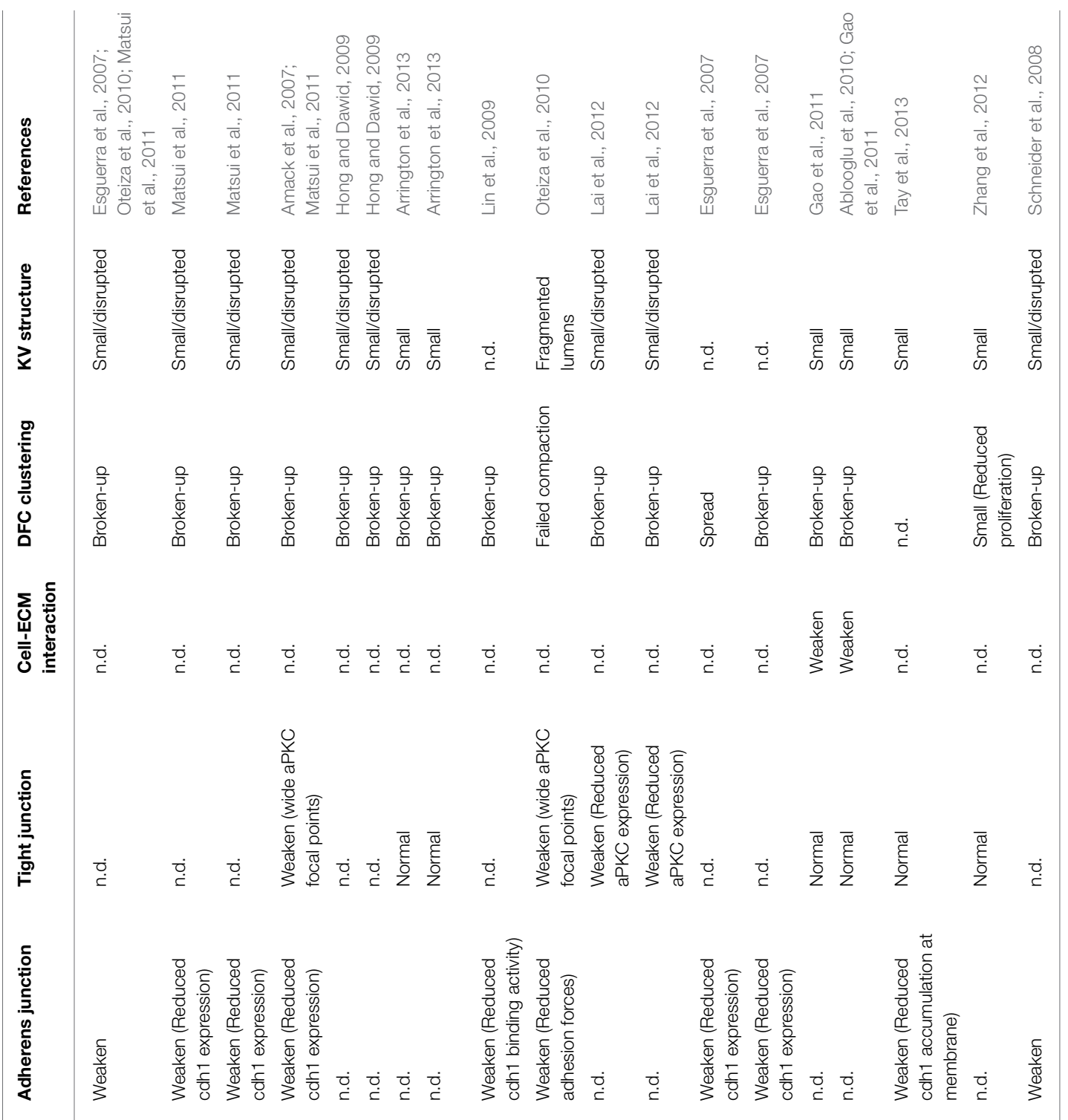

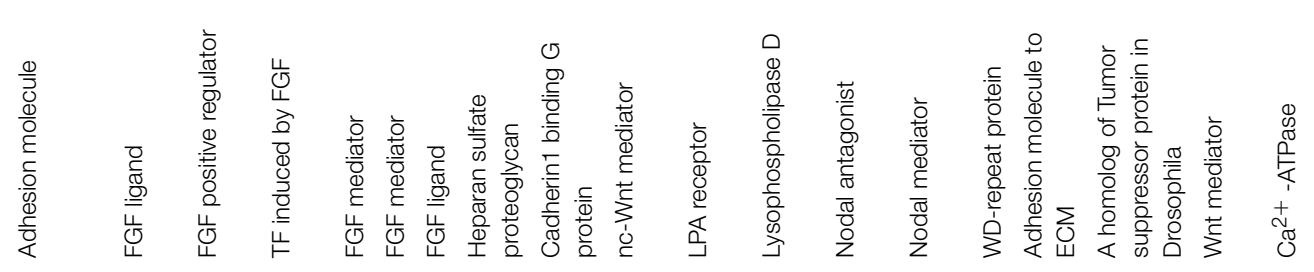

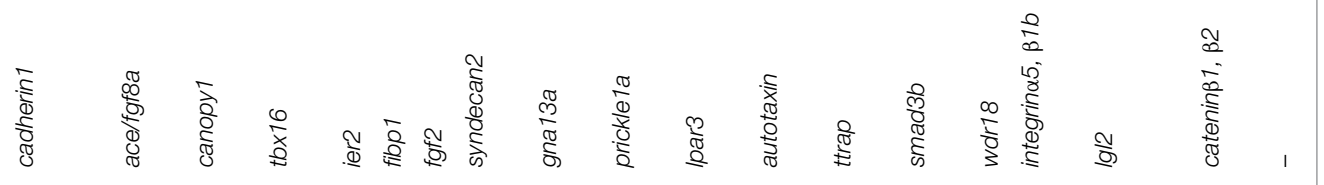


in these situations. These findings suggest that cooperation of several adhesive interactions is required to regulate DFC collectivity. Therefore, it will be important to characterize cooperative effects of adhesive interactions on cell collectivity regulation during collective DFC migration.

\section{Genes Essential for DFC Collectivity}

Many genes have been reported to be involved in the regulation of DFC collectivity, as listed in Table 1. As stated above, genes related to FGF and to Nodal signaling can function as regulators of DFC collectivity. In addition, knockdown of prickle1a, a noncanonical Wnt (nc-Wnt) regulator, leads to failure to compact the DFC cluster during late-gastrulation stages (9-10 hpf) (Table 1) (Oteiza et al., 2010), suggesting that nc-Wnt signaling also regulates cell collectivity. This idea is supported by data from the direct single-cell force spectroscopy measurement of adhesion properties between a pair of DFCs, which showed that the adhesion force between DFCs isolated from embryo depleted of prickle1a is reduced as compared to wildtype DFCs (Oteiza et al., 2010). This method is very good for measuring cell adhesion forces at the single cell level, while physiological interactions are disrupted during cell preparation. It would be of great interest to develop a method to measure physiological cell adhesion forces within living zebrafish embryos in the near future.

\section{Identification of Genes Required for DFC Collectivity Formation in Future}

A DFC-specific gene knockdown approach in which morpholino is injected into the yolk of embryos at the 256-512 cell stages has greatly contributed to our understanding of how DFC collectivity is regulated (this review), how DFC clustering occurs and whether DFC/KV morphogenesis is required for the establishment of left-right patterning (Amack and Yost, 2004; Amack et al., 2007; Matsui and Bessho, 2012). We therefore believe that this approach will be important to identify new genes required for DFC collectivity. However, very recently, Kok et al. have reported a problem for morpholino-induced knockdown approach (Kok et al., 2015). Approximately $80 \%$ of phenotypes induced by morpholinos are not observed in mutant embryos, indicating that morpholinos highly induce off-target effects; moreover, these problems are shared with other antisense technologies. It is thus recommended that only morpholinos that recapitulate the respective phenotypes seen in mutant embryos should be applied for ancillary analyses. As the DFC-specific gene knockdown approach is based on morpholino-induced knockdown, here is a good opportunity to consider whether we can apply this approach to investigate how DFC collectivity is regulated in future.

As this approach has similarities to the conditional knockout strategy, it is best to evaluate whether DFC-specific gene knockdown recapitulates the phenotypes caused in the DFCspecific conditional knockout zebrafish. Although insertion of loxP site into a zebrafish genome by genome editing technologies such as TALEN and CRISPER/Cas9 systems has recently been successful (Bedell et al., 2012; Chang et al., 2013), a method generating conditional knockout zebrafish has not yet been developed. Even though such a method is likely to be developed in the near future, we feel that it would be difficult to apply it to understand the roles of genes in DFC/KV formation.

In general, conditional gene knockout is carried out by using a Cre/loxP system. The expression of Cre recombinase is induced by a tissue/organ-specific promoter and Cre protein modulates the genome at the specific insertion site of loxP. Thus, it takes time to observe the signs of Cre/loxP recombination. For instance, in the case of conditional knockout mice, the signs of Cre/loxP recombination are detected an average of $24 \mathrm{~h}$ after the induction (Hayashi and McMahon, 2002; Chen et al., 2007). In the case of DFCs, however, collective DFC migration starts at almost the same time as the DFC specification (evident by the expression of early DFC marker genes, sox32 and sox17) and finishes just $3 \mathrm{~h}$ (Oteiza et al., 2008; Matsui and Bessho, 2012). It has been suggested that $\mathrm{Cre} /$ loxP recombination may not occur simultaneously with collective DFC migration without applying much faster recombination methods.

Because the DFC-specific gene knockdown approach is absolutely indispensable to provide new insights into collective DFC migration, KV formation, KV ciliogenesis, and leftright patterning in the near future, a guideline for DFCspecific gene knockdown is needed. We therefore propose one based on guidelines reported by Eisen and Smith (2008) and Kok et al. (2015). First, as with standard morpholinobased knockdown approaches, validate morpholino-induced phenotypes and compare them to those of the mutant. If the mutant is not available, generate one for the gene of interest and make sure of the morpholino-induced phenotypes by comparison. Second, inject the morpholino tagged with fluorescein or lissamine into the yolk of embryos at 256-512 cell stages (2.5-2.75 hpf), and select embryos in which morpholino has been correctly delivered into the DFCs. As an important control, also inject the same morpholino into the yolk of embryos at the sphere-dome stages (4-4.3 hpf) (Amack and Yost, 2004; Amack et al., 2007). Third, perform a rescue experiment by coinjecting morpholino and mRNA into the yolk of embryos at 256-512 cell stages (Matsui and Bessho, 2012).

\section{Dynamics of Collective DFC Migration}

During epiboly (6-10 hpf), the EVL cells tightly bind to the yolk syncytial layer (YSL) and purse string contraction of the actin in YSL drives the migration of EVL toward the vegetal pole (Lepage and Bruce, 2010; Lee, 2014). Because a subset of DFCs is linked to EVL through the formation of tight junction, it has been suggested that DFC-EVL tight junction is important to mediate the vegetal migration of DFCs during epiboly (Figures 1, 2). However, live imaging has revealed that DFCs located at the leading edge side of the cluster frequently generate cell protrusions such as filopodia and lamellipodia toward the vegetal pole (Ablooglu et al., 2010 and see also Figure 2). These findings suggest that, in addition to passive migration mediated by DFC-EVL tight junction, DFCs have the potential to migrate themselves toward the vegetal pole.

In collective cell migration of endothelial cells, the cells behave dynamically. For instance, a follower cell sometimes moves to the leading edge and becomes a leader cell (Jakobsson et al., 2010; Arima et al., 2011; Rorth, 2012), suggesting that cell behaviors are highly variable in individuals and positions, and 

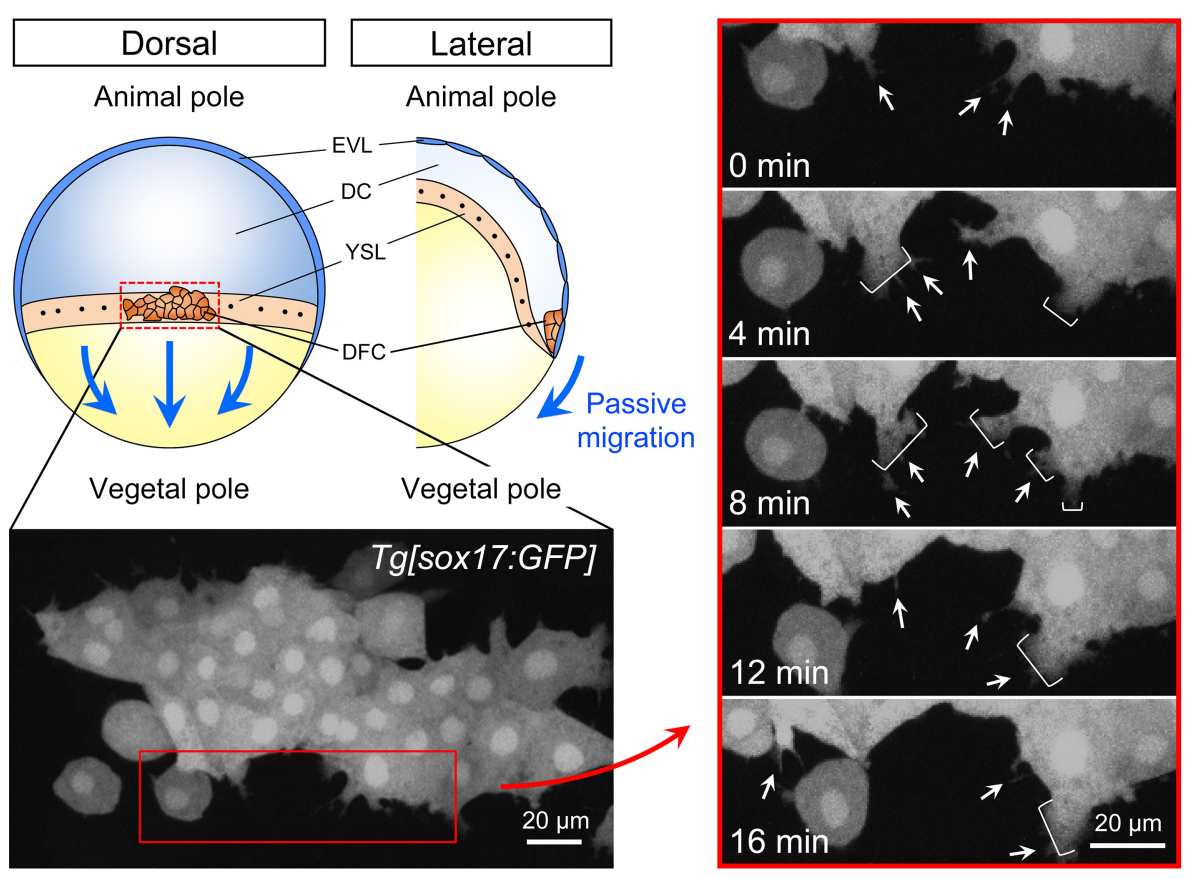

FIGURE 2 | Cell dynamics during collective DFC migration. Depending on the formation of tight junction between DFC and EVL, directed migration of the DFC cluster toward the vegetal pole passively occurs (blue arrows). EVL, enveloping layer; DC, deep cells; YSL, yolk syncytial layer; DFC, dorsal forerunner cells. Time-lapse images from 0 to 16 min (4 min intervals) in the control Tg[sox17:GFP] embryo (left panels).
Dorsal view, anterior to the top. Scale bar: $20 \mu \mathrm{m}$. As shown previously (Ablooglu et al., 2010), GFP-positive DFCs at the leading edge frequently generate filopodium (arrow) and lamellipodium (bracket) (right panels). These findings suggest that, in addition to the passive migration through the formation of DFC-EVL tight junction, DFCs have a potential to migrate toward the vegetal pole. that cell identities as leaders and followers can be changeable during collective cell migration. If individual DFCs behave dynamically during collective DFC migration, signal activities, which regulate cell collectivity and/or migration, are different among individual DFCs and change from time to time even in an individual DFC. Therefore, precise observation of individual DFC behaviors, adhesive properties, and signal activities in living zebrafish embryos will be important to understand mechanisms of collective DFC migration.

\section{Conclusion and Outlook}

Many studies using zebrafish as a model system of vertebrate development have provided us with new insights into how the laterality organ (KV) breaks left-right symmetry. Focusing on cell collectivity regulation during $\mathrm{KV}$ organogenesis, we now understand the importance and the regulatory mechanism of cell collectivity in these processes. Furthermore, we show that cell adhesive properties change during collective DFC migration, indicating that multicellular tissues/organs are more

\section{References}

Ablooglu, A. J., Tkachenko, E., Kang, J., and Shattil, S. J. (2010). Integrin alphaV is necessary for gastrulation movements that regulate vertebrate body asymmetry. Development 137, 3449-3458. doi: 10.1242/dev. 045310 dynamic than previously thought. Despite this substantial progress, many important questions remain. For instance, how do collective cell dynamics contribute to generating functional organs? How does the pairing of tight junction change? Are adherens junctions, tight junctions, and cell-ECM interaction coordinated? Does mechanical force contribute to collective DFC migration? Does collective DFC migration have analogy with other collective cell migrations seen in normal development, wound repair, and cancer invasion? It is of great interest to fill in these gaps to further clarify the regulatory mechanisms and importance of collective cell migration during organogenesis.

\section{Acknowledgments}

We are grateful to the Ministry of Education, Culture, Sports, Science and Technology (MEXT), the Uehara Memorial Foundation, the Nakajima Foundation and the Mochida Memorial Foundation for past and current support.

Amack, J. D., Wang, X., and Yost, H. J. (2007). Two T-box genes play independent and cooperative roles to regulate morphogenesis of ciliated Kupffer's vesicle in zebrafish. Dev. Biol. 310, 196-210. doi: 10.1016/j.ydbio.2007.05.039

Amack, J. D., and Yost, H. J. (2004). The T box transcription factor no tail in ciliated cells controls zebrafish left-right asymmetry. Curr. Biol. 14, 685-690. doi: 10.1016/j.cub.2004.04.002 
Arima, S., Nishiyama, K., Ko, T., Arima, Y., Hakozaki, Y., Sugihara, K., et al. (2011). Angiogenic morphogenesis driven by dynamic and heterogeneous collective endothelial cell movement. Development 138, 4763-4776. doi: 10.1242/dev.068023

Arrington, C. B., Peterson, A. G., and Yost, H. J. (2013). Sdc2 and Tbx16 regulate Fgf2-dependent epithelial cell morphogenesis in the ciliated organ of asymmetry. Development 140, 4102-4109. doi: 10.1242/dev. 096933

Babb, S. G., Barnett, J., Doedens, A. L., Cobb, N., Liu, Q., Sorkin, B. C., et al. (2001). Zebrafish E-cadherin: expression during early embryogenesis and regulation during brain development. Dev. Dyn. 221, 231-237. doi: 10.1002/dvdy.1132

Babb, S. G., and Marrs, J. A. (2004). E-cadherin regulates cell movements and tissue formation in early zebrafish embryos. Dev. Dyn. 230, 263-277. doi: 10.1002/dvdy.20057

Batlle, E., Sancho, E., Franci, C., Dominguez, D., Monfar, M., Baulida, J., et al. (2000). The transcription factor snail is a repressor of E-cadherin gene expression in epithelial tumour cells. Nat. Cell Biol. 2, 84-89. doi: $10.1038 / 35000034$

Bedell, V. M., Wang, Y., Campbell, J. M., Poshusta, T. L., Starker, C. G., Krug, R. G. II., et al. (2012). In vivo genome editing using a high-efficiency TALEN system. Nature 491, 114-118. doi: 10.1038/nature11537

Blum, M., Feistel, K., Thumberger, T., and Schweickert, A. (2014). The evolution and conservation of left-right patterning mechanisms. Development 141, 1603-1613. doi: 10.1242/dev.100560

Cano, A., Perez-Moreno, M. A., Rodrigo, I., Locascio, A., Blanco, M. J., del Barrio, M. G., et al. (2000). The transcription factor snail controls epithelialmesenchymal transitions by repressing E-cadherin expression. Nat. Cell Biol. 2, 76-83. doi: 10.1038/35000025

Chang, N., Sun, C., Gao, L., Zhu, D., Xu, X., Zhu, X., et al. (2013). Genome editing with RNA-guided Cas9 nuclease in zebrafish embryos. Cell Res. 23, 465-472. doi: $10.1038 / \mathrm{cr} .2013 .45$

Chen, M., Lichtler, A. C., Sheu, T. J., Xie, C., Zhang, X., O'Keefe, R. J., et al. (2007). Generation of a transgenic mouse model with chondrocyte-specific and tamoxifen-inducible expression of Cre recombinase. Genesis 45, 44-50. doi: 10.1002/dvg.20261

Choksi, S. P., Lauter, G., Swoboda, P., and Roy, S. (2014). Switching on cilia: transcriptional networks regulating ciliogenesis. Development 141, 1427-1441. doi: 10.1242/dev.074666

Compagnon, J., Barone, V., Rajshekar, S., Kottmeier, R., Pranjic-Ferscha, K., Behrndt, M., et al. (2014). The notochord breaks bilateral symmetry by controlling cell shapes in the zebrafish laterality organ. Dev. Cell. 31, 774-783. doi: 10.1016/j.devcel.2014.11.003

Cooper, M. S., and D'Amico, L. A. (1996). A cluster of noninvoluting endocytic cells at the margin of the zebrafish blastoderm marks the site of embryonic shield formation. Dev. Biol. 180, 184-198. doi: 10.1006/dbio.1996.0294

Eisen, J. S., and Smith, J. C. (2008). Controlling morpholino experiments: don't stop making antisense. Development 135, 1735-1743. doi: 10.1242/dev.001115

Esguerra, C. V., Nelles, L., Vermeire, L., Ibrahimi, A., Crawford, A. D., Derua, R., et al. (2007). Ttrap is an essential modulator of Smad3-dependent nodal signaling during zebrafish gastrulation and left-right axis determination. Development 134, 4381-4393. doi: 10.1242/dev.000026

Essner, J. J., Amack, J. D., Nyholm, M. K., Harris, E. B., and Yost, H. J. (2005). Kupffer's vesicle is a ciliated organ of asymmetry in the zebrafish embryo that initiates left-right development of the brain, heart and gut. Development 132, 1247-1260. doi: 10.1242/dev.01663

Essner, J. J., Vogan, K. J., Wagner, M. K., Tabin, C. J., Yost, H. J., and Brueckner, M. (2002). Conserved function for embryonic nodal cilia. Nature 418, 37-38. doi: $10.1038 / 418037 a$

Friedl, P., and Gilmour, D. (2009). Collective cell migration in morphogenesis, regeneration and cancer. Nat. Rev. Mol. Cell Biol. 10, 445-457. doi: $10.1038 / \mathrm{nrm} 2720$

Friedl, P., Locker, J., Sahai, E., and Segall, J. E. (2012). Classifying collective cancer cell invasion. Nat. Cell Biol. 14, 777-783. doi: 10.1038/ncb2548

Gao, W., Xu, L., Guan, R., Liu, X., Han, Y., Wu, Q., et al. (2011). Wdr18 is required for Kupffer's vesicle formation and regulation of body asymmetry in zebrafish. PLoS ONE 6:e23386. doi: 10.1371/journal.pone.0023386

Hajra, K. M., Chen, D. Y., and Fearon, E. R. (2002). The SLUG zinc-finger protein represses E-cadherin in breast cancer. Cancer Res. 62, 1613-1618.
Harrington, M. J., Hong, E., Fasanmi, O., and Brewster, R. (2007). Cadherinmediated adhesion regulates posterior body formation. BMC Dev. Biol. 7:130. doi: 10.1186/1471-213X-7-130

Hartsock, A., and Nelson, W. J. (2008). Adherens and tight junctions: structure, function and connections to the actin cytoskeleton. Biochim. Biophys. Acta 1778, 660-669. doi: 10.1016/j.bbamem.2007.07.012

Hayashi, S., and McMahon, A. P. (2002). Efficient recombination in diverse tissues by a tamoxifen-inducible form of cre: a tool for temporally regulated gene activation/inactivation in the mouse. Dev. Biol. 244, 305-318. doi: 10.1006/dbio.2002.0597

Hirokawa, N., Tanaka, Y., and Okada, Y. (2012). Cilia, KIF3 molecular motor and nodal flow. Curr. Opin. Cell Biol. 24, 31-39. doi: 10.1016/j.ceb.2012.01.002

Hirokawa, N., Tanaka, Y., Okada, Y., and Takeda, S. (2006). Nodal flow and the generation of left-right asymmetry. Cell 125, 33-45. doi: 10.1016/j.cell.2006.03.002

Hong, S. K., and Dawid, I. B. (2009). FGF-dependent left-right asymmetry patterning in zebrafish is mediated by Ier2 and Fibp1. Proc. Natl. Acad. Sci. U.S.A. 106, 2230-2235. doi: 10.1073/pnas.0812880106

Ishikawa, H., and Marshall, W. F. (2011). Ciliogenesis: building the cell's antenna. Nat. Rev. Mol. Cell Biol. 12, 222-234. doi: 10.1038/nrm3085

Jakobsson, L., Franco, C. A., Bentley, K., Collins, R. T., Ponsioen, B., Aspalter, I. M., et al. (2010). Endothelial cells dynamically compete for the tip cell position during angiogenic sprouting. Nat. Cell Biol. 12, 943-953. doi: 10. $1038 /$ ncb2 103

Kerstetter, A. E., Azodi, E., Marrs, J. A., and Liu, Q. (2004). Cadherin-2 function in the cranial ganglia and lateral line system of developing zebrafish. Dev. Dyn. 230, 137-143. doi: 10.1002/dvdy.20021

Kok, F. O., Shin, M., Ni, C. W., Gupta, A., Grosse, A. S., van Impel, A., et al. (2015) Reverse genetic screening reveals poor correlation between morpholinoinduced and mutant phenotypes in zebrafish. Dev. Cell. 32, 97-108. doi: 10.1016/j.devcel.2014.11.018

Lai, S. L., Yao, W. L., Tsao, K. C., Houben, A. J., Albers, H. M., Ovaa, H. et al. (2012). Autotaxin/Lpar3 signaling regulates Kupffer's vesicle formation and left-right asymmetry in zebrafish. Development 139, 4439-4448. doi: 10.1242/dev.081745

Lee, S. J. (2014). Dynamic regulation of the microtubule and actin cytoskeleton in zebrafish epiboly. Biochem. Biophys. Res. Commun. 452, 1-7. doi: 10.1016/j.bbrc.2014.08.005

Lepage, S. E., and Bruce, A. E. (2010). Zebrafish epiboly: mechanics and mechanisms. Int. J. Dev. Biol. 54, 1213-1228. doi: 10.1387/ijdb.093028sl

Lin, F., Chen, S., Sepich, D. S., Panizzi, J. R., Clendenon, S. G., Marrs, J. A., et al. (2009). Galpha12/13 regulate epiboly by inhibiting E-cadherin activity and modulating the actin cytoskeleton. J. Cell Biol. 184, 909-921. doi: $10.1083 /$ jcb. 200805148

Matsui, T., and Bessho, Y. (2012). Left-right asymmetry in zebrafish. Cell Mol. Life Sci. 69, 3069-3077. doi: 10.1007/s00018-012-0985-6

Matsui, T., Thitamadee, S., Murata, T., Kakinuma, H., Nabetani, T., Hirabayashi, Y., et al. (2011). Canopy1, a positive feedback regulator of FGF signaling, controls progenitor cell clustering during Kupffer's vesicle organogenesis. Proc. Natl. Acad. Sci. U.S.A. 108, 9881-9886. doi: 10.1073/pnas. 1017248108

Melby, A. E., Warga, R. M., and Kimmel, C. B. (1996). Specification of cell fates at the dorsal margin of the zebrafish gastrula. Development 122, 2225-2237.

Nakamura, T., and Hamada, H. (2012). Left-right patterning: conserved and divergent mechanisms. Development 139, 3257-3262. doi: 10.1242/dev.061606

Niewiadomska, P., Godt, D., and Tepass, U. (1999). DE-cadherin is required for intercellular motility during drosophila oogenesis. J. Cell Biol. 144, 533-547. doi: $10.1083 /$ jcb.144.3.533

Oteiza, P., Koppen, M., Concha, M. L., and Heisenberg, C. P. (2008). Origin and shaping of the laterality organ in zebrafish. Development 135, 2807-2813. doi: 10.1242/dev.022228

Oteiza, P., Koppen, M., Krieg, M., Pulgar, E., Farias, C., Melo, C., et al. (2010). Planar cell polarity signalling regulates cell adhesion properties in progenitors of the zebrafish laterality organ. Development $137,3459-3468$. doi: 10.1242/dev.049981

Reig, G., Pulgar, E., and Concha, M. L. (2014). Cell migration: from tissue culture to embryos. Development 141, 1999-2013. doi: 10.1242/dev.101451

Rorth, P. (2009). Collective cell migration. Annu. Rev. Cell Dev. Biol. 25, 407-429. doi: 10.1146/annurev.cellbio.042308.113231 
Rorth, P. (2012). Fellow travellers: emergent properties of collective cell migration. EMBO Rep. 13, 984-991. doi: 10.1038/embor.2012.149

Schneider, I., Houston, D. W., Rebagliati, M. R., and Slusarski, D. C. (2008). Calcium fluxes in dorsal forerunner cells antagonize beta-catenin and alter left-right patterning. Development 135, 75-84. doi: 10.1242/dev.004713

Schober, M., Rebay, I., and Perrimon, N. (2005). Function of the ETS transcription factor yan in border cell migration. Development 132, 3493-3504. doi: $10.1242 / \mathrm{dev} .01911$

Siddiqui, M., Sheikh, H., Tran, C., and Bruce, A. E. (2010). The tight junction component claudin E is required for zebrafish epiboly. Dev. Dyn. 239, 715-722. doi: $10.1002 /$ dvdy.22172

Tay, H. G., Schulze, S. K., Compagnon, J., Foley, F. C., Heisenberg, C. P., Yost, H. J., et al. (2013). Lethal giant larvae 2 regulates development of the ciliated organ Kupffer's vesicle. Development 140, 1550-1559. doi: 10.1242/dev.087130

Trinh, L. A., and Stainier, D. Y. (2004). Fibronectin regulates epithelial organization during myocardial migration in zebrafish. Dev. Cell. 6, 371-382. doi: 10.1016/S1534-5807(04)00063-2

Warga, R. M., and Kane, D. A. (2007). A role for N-cadherin in mesodermal morphogenesis during gastrulation. Dev. Biol. 310, 211-225. doi: 10.1016/j.ydbio.2007.06.023
Weijer, C. J. (2009). Collective cell migration in development. J. Cell. Sci. 122, 3215-3223. doi: $10.1242 /$ jcs. 036517

Wilson, A. L., Shen, Y. C., Babb-Clendenon, S. G., Rostedt, J., Liu, B., Barald, K. F., et al. (2007). Cadherin-4 plays a role in the development of zebrafish cranial ganglia and lateral line system. Dev. Dyn. 236, 893-902. doi: 10.1002/dvdy.21085

Zhang, M., Zhang, J., Lin, S. C., and Meng, A. (2012). Beta-catenin 1 and betacatenin 2 play similar and distinct roles in left-right asymmetric development of zebrafish embryos. Development 139, 2009-2019. doi: 10.1242/dev.074435

Conflict of Interest Statement: The authors declare that the research was conducted in the absence of any commercial or financial relationships that could be construed as a potential conflict of interest.

Copyright (๔) 2015 Matsui, Ishikawa and Bessho. This is an open-access article distributed under the terms of the Creative Commons Attribution License (CC BY). The use, distribution or reproduction in other forums is permitted, provided the original author(s) or licensor are credited and that the original publication in this journal is cited, in accordance with accepted academic practice. No use, distribution or reproduction is permitted which does not comply with these terms. 